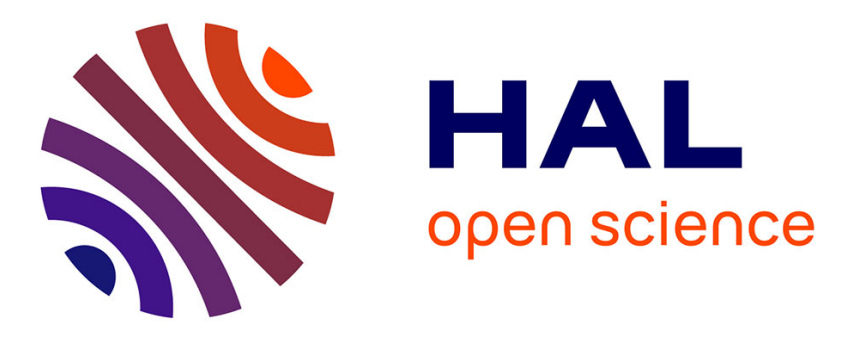

\title{
"Il est un meilleur roi que le roi d'Angleterre". Note sur la diffusion et la fonction d'une rumeur dans la paysannerie du Bordelais au XIIIe siècle
}

Frédéric Boutoulle

\section{- To cite this version:}

Frédéric Boutoulle. "Il est un meilleur roi que le roi d'Angleterre". Note sur la diffusion et la fonction d'une rumeur dans la paysannerie du Bordelais au XIIIe siècle. Maïté Billoré; Myriam Soria. La rumeur au Moyen âge. Du mépris à la manipulation, Ve-XVe siècles, Presses Universitaires de Rennes, pp.279-291, 2011, 978-2-7535-1285-6. 10.4000/books.pur.119706 . hal-01475169

\author{
HAL Id: hal-01475169 \\ https://hal.science/hal-01475169
}

Submitted on 24 Nov 2017

HAL is a multi-disciplinary open access archive for the deposit and dissemination of scientific research documents, whether they are published or not. The documents may come from teaching and research institutions in France or abroad, or from public or private research centers.
L'archive ouverte pluridisciplinaire $\mathbf{H A L}$, est destinée au dépôt et à la diffusion de documents scientifiques de niveau recherche, publiés ou non, émanant des établissements d'enseignement et de recherche français ou étrangers, des laboratoires publics ou privés. 


\section{« Il est un meilleur roi que le roi d'Angleterre ». Note sur la diffusion et la fonction d'une rumeur dans la paysannerie du Bordelais au XIII ${ }^{\mathrm{e}}$ siècle}

\section{Frédéric Boutoulle}

En 1237, dans le procès verbal d'une enquête diligentée par le roi d'Angleterre et duc d'Aquitaine Henri III sur les excès commis par ses baillis en Entre-deux-Mers bordelais, un passage rapporte des accusations contre le sénéchal Henri de Trubleville accusé de se substituer au roi en nommant lui-même un sous-sénéchal ${ }^{1}$ :

«Cependant comme un sous-sénéchal est nommé contrairement à l'antique coutume de la terre, celui qui désigna un sénéchal pour le remplacer [c'est à dire le sénéchal lui-même] s'érigea en roi, ainsi qu'il le dit lui-même à maintes reprises comme beaucoup de bons hommes l'ont entendu; [qu'en plus ] il était un meilleur roi que le seigneur roi d'Angleterre, qu'il n'en ferait pas davantage en sa faveur pour rien ; et il dit davantage encore de plus honteux qu'il est digne de rapporter $»^{2}$.

Cette phrase, pas toujours très claire, véhicule une autre accusation selon laquelle le sénéchal aurait dévalorisé le souverain en se considérant meilleur que lui. À notre connaissance, il s'agit de la première mention d'une critique contre la personne du monarque dans la documentation régionale, avant la grande révolte de la fin des années 1240 focalisée contre le lieutenant du roi, son beau-frère Simon de Monfort. À ce titre, parce qu'ils peuvent constituer une forme d'expression de ce que d'aucun appellent l'opinion publique, ces propos méritent d'être examinés, ne serait-ce que parce que la critique du pouvoir en général pose le problème de l'existence d'un « espace public », au sens habermassien ${ }^{3}$. Au même moment ou peu s'en faut, des pamphlets circulant contre Blanche de Castille entre 1228 et 1231, accusée de prévarication et d'avoir de mauvaises mœurs, sont considérés comme une des premières expressions de l'opinion publique en France, «par des manifestations ouvertes, des jugements populaires collectifs, spontanés ou non, sur les affaires du royaume et le comportement des gouvernants ${ }^{4} \gg$. Dans une veine plus imagée, on connaît aussi l'opinion collectif des Gascons coalisés dans la ligue de Pons vis-à-vis des sentiments d'Henri III à leur endroit, rapporté par un habitant de la Rochelle dans une lettre au roi de France en 1241 : « ils font ce qu'ils

\footnotetext{
${ }^{1}$ Il existe deux versions de cette enquête. La première, en latin, est transcrite avant 1270 dans le Petit cartulaire de La Sauve-Majeure (Bibliothèque municipale de Bordeaux, ms 770, pp. 126-135, dorénavant PCSM), partiellement éditée dans Gallia Christiana, t.II, col. 289-293). Une version gasconne a été transcrite dans un registre du XIV siècle, Privileyges de la terra de Entre dos Mars (Bibliothèque municipale de Bordeaux, ms 363) et éditée par J. Delpit, "Coutumes et privilèges de l'Entre-deux-Mers», Archives Historiques du département de la Gironde, t. III, Bordeaux, 1861-1862, pp. 101-127.

${ }^{2}$ PCSM, p. 130 : Cum tamen subsenescalcus contra antiquam consuetudinem terre datur, set regem se innuit qui sibi substituit senescalcum, sicut idem multociens multis bonis viris audientibus dixit, quod melius erat rex quam dominus rex Anglie, et non faceret pro ipso quantum pro nichilo, immo et turpius dixit quam sit relatu dignum. Privileyges de la terra d'Entre-dos-Mars, p. 122 : Empero cum lo sotz senescaut es donat, contra la costuma antiqua de la terra, aquet qui constituit luy senescaut se emmys Reys ayssi cum es dischut, mantas beguadas, mans bons homes audiens, que melhor era rey que no lo senhor rey d'Anglaterra et no faria per luy quant nos res ans dischut plus layh que no es digna causa de diser. Nous tenons à remercier Xavier Ravier et Patrick Henriet pour leurs remarques.

${ }^{3}$ J. HABERMASS, L'espace public: archéologie de la publicité comme dimension constitutive de la société bourgeoise, 1963 ; S. HABER, «Quelques mots pour historiciser L'espace public de Habermas », dans L'espace public au Moyen Age, Journée d'étude du LAMOP, 31 mai 2005, s.d. P. Boucheron.

${ }^{4}$ J. Le GoFF, Saint Louis, Paris, 1996, p. 104.
} 
veulent car, pour le roi des Anglais, que ce soit à Bordeaux ou à Bayonne, ce qu'ils font ne vaut pas un œuf $»^{5}$. Le contexte est donc à l'émergence d'une opinion critique sur le souverain.

Les propos d'Henri de Trubleville sur le roi Henri III peuvent être considérés comme une information à la limite de la rumeur ou inversement. En effet, ils n'ont pas été consignés directement de la bouche des «bons hommes ». Leur origine n'est pas bien connue, puisque ceux qui déposent devant les enquêteurs sont présentés derrière le terme de "jurés» ou l'expression « les plus anciens de cette terre », jamais comme des « bons hommes ». Il est vrai que parmi les jurés, dont on verra qu'ils se font fréquemment les porte-parole des élites rurales, comptent probablement ceux que l'on appelle communément des «bons hommes ». Pour autant, et on tient à le souligner, l'information n'arrive qu'indirectement aux oreilles des enquêteurs. Parallèlement, la même enquête révèle d'autres rumeurs pour lesquelles l'attitude des commissaires royaux est différente. Ce cas permet donc de répondre à quelques unes des interrogations qui nous réunissent sur les circonstances dans lesquelles naît une rumeur au XIII ${ }^{\mathrm{e}}$ siècle, sur ses canaux de diffusion, sur l'attitude des autorités à son encontre, sur l'usage qu'en font les acteurs sociaux comme sur celui des historiens qui cherchent, grâce à elle, à mieux appréhender les groupes sociaux.

Nous nous arrêterons dans un premier temps sur les auteurs supposés de ces propos afin de comprendre le ressentiment qu'ils focalisent. Puis, en nous attachant au traitement de la critique faite au roi dans cette enquête, il s'agira de restituer toute la singularité de cette rumeur et de réfléchir à la notion de rumeur interdite. Nous terminerons avec ses émetteurs, le groupe des « bons hommes » et les enjeux sociaux qu'elle mobilise chez eux.

\section{Les cibles du ressentiment des jurés}

Ces propos sont attribués au sénéchal. Qui est-il ? Pourquoi avec le sous-sénéchal focalisent-ils autant de ressentiments ?

Henri de Trubleville est alors sénéchal de Gascogne depuis le 23 mai 1234 pour la deuxième fois, puisqu'il a occupé les mêmes fonctions entre le 19 octobre 1227 et le $1^{\text {er }}$ juillet $1231^{6}$. Il appartient à une famille d'origine normande, dont le siège, Trubleville est situé près de Rouen. Sa famille est aussi possessionnée en Angleterre, puisque son oncle Ralf de Trubleville, sheriff du Northamptonshire en 1223, a obtenu les seigneuries de Brockhampton (Herefordshire) et de Combrook (Warwickshire) ${ }^{7}$. Les rôles de la chancellerie anglaise livrent beaucoup de données sur Henri entre 1228 et 1238, y compris pendant les trois années séparant ses deux sénéchalats, pendant lesquelles il obtient du roi quelques seigneuries anglaises ainsi que les îles de Jersey et Guernesey ${ }^{8}$. Le 25 mai 1234, soit deux jours après sa

\footnotetext{
${ }^{5}$ L. DELISLE, « Mémoire sur une lettre inédite adressée à la reine Blanche par un habitant de la Rochelle », Bibliothèque de l'École des Chartes, 1856, vol. 17, $\mathrm{n}^{\circ} 1$, p. 527 : Qui omnes unanimiter dixerunt quod, si subjugati fuerint Gallicis, destruhentur: nunc enim terra sua est, et faciunt quicquid volunt, quia pro rege Anglorum, etiam apud Burdegalam et Bayonam, non faciunt valens ovum.

${ }^{6}$ W.-W. SHIRLEY ed. Royal and other historical letters illustrative of the reign of Henry III, Londres, 1862, p. 399-400. H. de Trubleville redevient sénéchal une troisième fois, entre le 28 novembre 1238 et avant le 22 septembre 1241 .

${ }^{7}$ Calendar of Patent rolls preserved in the Public Record office, Henry III, 1232-1247, p. 33 ; S. D. CHURCH, The Household Knights of King John, Cambridge, 1999, p. 119-120.

${ }^{8}$ Calendar of Patent rolls preserved in the Public Record office, Henry III, 1232-1247, p. 25, 33 ; J. HAVERT, "Série chronologique des gardiens et seigneurs des îles normandes (1198-1468) », Bibliothèque de l'Ecole des Chartes, 1876, 37, p. 194.
} 
nomination, Henri III assigne à douze de ces créanciers gascons, la perception de revenus publics à Bordeaux pendant cinq ans jusqu'au remboursement de la somme de 6000 marcs ${ }^{9}$.

En janvier 1237, Henri de Trubleville est sénéchal depuis bientôt trois ans et, si l'on en croit les dépositions recueillies par les enquêteurs, toujours soucieux d'obtenir de l'argent en pressurant ses administrés, particulièrement en Entre-deux-Mers, cette fraction importante du domaine ducal s'étendant à l'est de Bordeaux, entre la Garonne, la Dordogne et le bourg de la Sauve-Majeure et où l'habitat est encore majoritairement dispersé. On se plaint des tailles qu'il lève indument sur les paroisses de cette région, des vols de volailles qu'il fait capturer au filet, des humiliations que ses sergents font subir aux prêtres et aux veuves, etc. Mais dans le passage qui nous intéresse, on lui reproche de «se faire roi en désignant un sénéchal pour le remplacer » et de s'en être vanté. Cela fait référence à la désignation par ce même Trubleville d'un sous-sénéchal.

La fonction de sous-sénéchal est récente. À notre connaissance, elle apparaît pour la première fois dans cette enquête, soit une quinzaine d'années avant 1253, date à laquelle JeanPaul Trabut-Cussac plaçait le partage des fonctions du sénéchal de Gascogne entre plusieurs sénéchaux ${ }^{10}$. Mais déjà, en 1228 , Trubleville avait tenté d'obtenir du roi la nomination d'un autre sénéchal, ce à quoi Henri III s'était opposé, se contentant de lui annoncer sa prochaine venue $^{11}$. L'homme a donc de la suite dans les idées. Mais alors qu'après 1250, lorsque la fonction est habituelle, les sous-sénéchaux sont des sénéchaux responsables de territoires ayant été adjoints au ressort du sénéchal de Gascogne (Bigorre, Périgord, Agenais), ce n'est pas le cas ici. Les activités du sous-sénéchal évoquées à plusieurs reprises dans l'enquête correspondent à celles des lieutenants des années 1250 , ces auxiliaires du sénéchal ayant à se substituer à lui pendant ses fréquentes absences afin d'assurer la continuité de son autorité (comme la tenue des assises). De fait, comme plus tard les lieutenants, les sous-sénéchaux de l'enquête sont nommés par le sénéchal et rémunérés par lui : ils ne sont donc responsables que devant le sénéchal, qui répond de leurs actes devant le roi-duc.

Les seuls agissements documentés sont limités aux extorsions qu'on reproche au soussénéchal. "Le sénéchal ou le sous-sénéchal, quand ils ont des amis à Bordeaux ou ailleurs, appellent les pauvres paysans, pour qu'avec leurs chars et leurs bœufs, ils portent les poutres et les tuiles des maisons, ainsi que le bois de chauffage $»^{12}$. Le sous-sénéchal, le sénéchal et les prévôts multiplient les aubergades sur les paysans. Les accusations sont plus précises pour deux paroisses. Dans celle de Lignan, le sous-sénéchal Arnaud Ramnou est accusé d'avoir extorqué deux fois la somme de soixante sous à la paroisse et de s'être fait indûment héberger avec une troupe de trente cavaliers ; dans la même paroisse, il est reproché au sous-sénéchal Rostand Soler d'avoir, avec un prévôt, mis à rançon un pèlerin de Saint-Jacques de Compostelle pour la somme de trois cents sous ${ }^{13}$. Dans les deux paroisses voisines de Camblanes et Quinsac, il est reproché au sous-sénéchal Arnaud Ramnou d'avoir extorqué quatre livres dans la première et, dans la seconde, sept livres plus un hébergement d'une

\footnotetext{
${ }^{9}$ Calendar of Patent rolls preserved in the Public Record office. Henry III, 1232-1247, p. 49.

${ }^{10}$ J.-P. TRABUT-CuSSAC, L'administration anglaise en Gascogne sous Henri III et Edouard I ${ }^{e r}$ de 1254 à 1307 , Genève, 1972 pp. 175-191.

${ }^{11}$ Close rolls of the reign of Henry III preserved in the Public Record Office, I, 1227-1231, p.112-113 (1228) :

Super eo quod nos rogastis ut loco vestro alium senescallum durantibus treugis substitueremus.

12 PCSM, p. 130, Cum senescalcus vel subsenescalcus habet amicum burdegalensium, vel alibi compelluntur miseri agricole, cum curribus et bobus portare tigna domorum et tegulas et ligna ad ignem.

${ }^{13}$ PCSM, p. 132.
} 
valeur de soixante sous. Il est aussi reproché au sous-sénéchal de conduire l'ost à la guerre à l'instar du sénéchal ${ }^{14}$.

Des deux sous-sénéchaux cités dans l'enquête, nous ne savons rien du premier, Arnaud Ramnou, sur ses origines familiales ou géographiques. Le second est loin d'être un inconnu. Rostand Soler est un des plus puissants bourgeois de Bordeaux que l'on suit depuis la fin des années dix du XIII ${ }^{\mathrm{e}}$ siècle. C'est un négociant en vin, important prêteur sur gage, notamment auprès du roi. Henri III le dédommage en lui confiant la charge de prévôt de Belin, dans les Landes du Bordelais (1220), puis les revenus de ce château en 1224. Il fait partie des créanciers du sénéchal Henri de Trubleville recevant une assignation de rente le 25 mai 1234. En 1237-1238, il est maire de Bordeaux. En 1242, Rostand est le premier bordelais à atteindre la distinction de sénéchal de Gascogne.

Si les sous-sénéchaux focalisent un fort ressentiment, ce n'est pas seulement en raison de leurs exactions. Comme eux, les prévôts exigent des hébergements considérés indus, sur les paysans et les paroisses ; ils rançonnent pour des montants équivalents ou supérieurs. Les sous-sénéchaux ne sont donc pas plus violents ou plus avides que les autres baillis du roi. Ce qui explique le ressentiment à leur encontre c'est d'abord que la fonction de sous-sénéchal n'est pas coutumière puisque cet ersatz de sénéchal «a été donné à l'encontre de la coutume de cette terre ${ }^{15}$. Aussi, contrairement au sénéchal, le sous-sénéchal ne peut pas avoir prêté serment en début de charge ni reçu en retour celui des habitants.

Il faut aussi, pour expliquer le ressentiment des habitants de 1'Entre-deux-Mers contre le sous-sénéchal, mettre en avant l'appartenance de Rostand Soler à une bourgeoisie et une municipalité qui prend ses aises sur leur région. Un passage de l'enquête dénonce justement la passivité et la tiédeur du sénéchal face aux agissements des plus puissants milites et ceux des bourgeois de Bordeaux, associés ici dans une commune réprobation, qu'il laisse injurier ou molester des milites moins puissants et les paysans du roi ${ }^{16}$. D'autres sources rendent compte des efforts de la municipalité de Bordeaux durant les premières décennies du XIII ${ }^{\mathrm{e}}$ siècle pour construire sa banlieue, notamment vers l'Entre-deux-Mers, avec des résultats mitigés; en 1222 , le roi interdit aux prud'hommes de Bordeaux de lever une taille de trois sous par maison en Entre-deux-Mers, car décidée sans son consentement ni celui du sénéchal ${ }^{17}$.

Ainsi, par son appartenance à une municipalité dont on se méfie en Entre-deux-Mers, parce qu'il exerce des fonctions baillivales en dehors d'un cadre coutumier et enfin parce qu'il n'est pas lié par un serment, Rostand Soler focalise un ressentiment probablement plus aigu que celui que suscitent les autres baillis ducaux.

\section{Le traitement dans l'enquête des propos attribués au sénéchal}

\footnotetext{
14 PCSM, p. 130. Subsenescalcus eque magnum, exercitum ducit quem ad modis senescalcis, vel interdum maiorem

${ }^{15}$ PCSM, p. 130, Cum tamen subsenescalcus contra antiquam consuetudinem terre datur, ( ...) Tempore Henrici et Ricardi, quod unicus esset senescalcus tocius terre regis in archiepiscopatu Burdegalensi et Auxitano et unicus prepositus Inter duo Maria, cum duobus mandatoribus supradictis, nec fiebat substitutio senescalci.

${ }^{16}$ PCSM, p. 131, Item propter incuriam et tepiditatem senescalci, milites potentes vel burgenses Burdegale non conqueruntur ei vel alii ballivo ipsius, cum dicunt minus potentes milites vel agricolas domni regis injurias sibi fecisse.

${ }^{17}$ Patent rolls of the reign of Henri III preserved in the Public Record Office, vol. I. AD 1216-1225, pp. 354-355 (4 décembre1222), vos sine precepto nostro vel licencia senescalli nostri tallagium posuistis super homines nostros manentes Inter Duo Maria, exigentes de singulis mansionibus III solidos de moneta illa.
} 
Les propos attribués à Henri de Trubleville sont doublement singuliers, d'abord parce qu'il s'agit d'une critique faite au roi ensuite parce qu'ils sont, d'un point de vue lexicologique, traités de manière particulière.

Relevons d'abord dans la phrase cette assez nette gradation entre rumeur autorisée, celle que l'on peut divulguer, et rumeur interdite, que l'on juge indigne d'être consignée. Cette distinction n'est pas exceptionnelle, Xavier Nadrigny l'a par exemple bien soulignée dans son étude sur la rumeur et l'opinion publique à Toulouse à la fin du Moyen Âge ${ }^{18}$. Mais contrairement à ce cas, où la rumeur interdite, celle que l'on n'enregistre pas, touche à la personne du roi, dans notre cas, le curseur est déplacé.

Pourtant, l'enregistrement de cette rumeur critique ne se fait pas sans gène. Les propos du sénéchal ne sont pas «dits» par un ou des témoins identifiés. On précise seulement que ces propos ont été « entendus » par des tiers et que c'est le nombre des auditeurs qui en a fait la publicité. Cette mise à distance détonne dans le texte de l'enquête. Le reste du temps les enquêteurs consignent les «dits» de jurés, le plus souvent sous la forme d'une suite de dispositions d'ordre normatif ou de faits développés à la suite d'une phrase du genre «les jurés ont dit » ou « les jurés ont déposé », ce qui nous vaut d'avoir un procès verbal d'enquête expurgé de la parole des témoins et faisant la part belle au travail de réécriture des enquêteurs, l'anglais Hubert Hose et l'abbé de La Grâce-Dieu. De temps à autre émerge aussi la parole des enquêteurs à travers un pluriel : « il nous dit » à propos d'un témoin qu'il a fallu aller voir à son domicile ; « nous croyons » à propos de l'origine de dévolution de pouvoirs judicaires ${ }^{19}$. Une autre fois, l'irruption du pluriel raconte une enquête diligentée par les enquêteurs euxmêmes pour vérifier le bien fondé d'une autre rumeur : " nous avons spécialement enquêté sur la personne d'Arnaud Guilhem Bravion parce qu'il est souvent arrivé à nos oreilles qu'il était fréquemment diffamé par les baillis et c'est pourquoi nous avons interrogé sur cela les jurés de la paroisse de Sadirac, où il vit $»^{20}$. Nous n'avons relevé, en dehors de ce cas, qu'une seule autre tournure passive, creditur, à propos d'une extorsion du sous-sénéchal Rostand Soler dans la paroisse de Barsac, faite « croit-on, avec l'assentiment du sénéchal ${ }^{21}$.

Cet arrière-plan de formules destinées à identifier l'origine des informations recueillies dans cette enquête nous permet de mieux apprécier la singularité de la relation des propos attribués au sénéchal. Ils ne sont pas directement «dits» par un témoin identifié, pas davantage évoqués par les enquêteurs eux-mêmes. On se contente de faire savoir qu'ils ont été « entendus » par plusieurs auditeurs. Il y a visiblement une forme de retenue, une parole que l'on n'avoue pas. L'occultation de cette chaîne de parole montre que l'on est à la limite du dicible et du non dicible. C'est comme si personne ne tenait à endosser la responsabilité de

\footnotetext{
${ }^{18}$ X. NARDRIGNY, « Rumeur et opinion publique à Toulouse à la fin du Moyen Âge », Annales du Midi, 2009, vol. $121, \mathrm{n}^{\circ} 265$, p.132-133.

${ }^{19}$ PCSM, p. 129, De hiis inquam iusticiam concesserunt sibi pro pace tuenda, et ratione istorum excessuum fidantiam super omnes laicos cuiuscumque homines essent, ipse tamen domnus rex sicut credimus dedit postea vicarias suas paucis quibusdam militibus.

${ }^{20}$ PCSM, p. 132, De persona Arnaldus Willelmi Bravion specialiter inquisivimus quia frequenter apud aures nostras a ballivis regis super hoc diffamabatur et invenimus per illos qui juraverant de parrochia ipsius de Sadirac ubi ipse commoratur, scilicet Vitalem de la Barreira, et P. Oliver quod nichil violenter vel fraudulenter occupavit de terra regis, set verum est quod emit a quodam libero homine regis qui recessit in Hyspaniam terram suam salvo jure regis et dedit eam cuidam capelle in parrochia de Sadirac salvo iure regis.

${ }^{21}$ PCSM, p. 132, In parrochia de Barsac viculo qui appellatur Villa Centulli appropriavit sibi per violentiam Rostandus de Solario VII vel octo homines domni regis et constituit sibi tributarios suos singulos, in singulis $X$ solidos quod factum fuit infra $V$ annos et sicut creditur consilio et consensu senescalci Henrici.
} 
verbaliser officiellement une pareille affirmation. Ainsi, quoi que l'on se trouve face à une rumeur critique considérée comme " autorisée », les réserves que suscite son enregistrement trahissent la proximité de la limite avec une rumeur interdite. Ce type de précautions aide donc à mesurer les prévenances dont le roi fait l'objet au sein de la population du duché et la conscience que médire de lui est transgressif, on y reviendra.

\section{Diffusion et légitimation de la rumeur auprès des élites rurales}

Les boni homines au sein desquels se diffuse l'information ou la rumeur correspondent aux élites rurales dont il est souvent question dans l'enquête et que la chancellerie anglaise reconnaît plus volontiers sous l'expression de "prud'hommes de l'Entre-deux-Mers » (probi homines). C'est à eux que Jean sans Terre a confirmé les libertés et privilèges par une charte en $1214^{22}$. Les prud'hommes de l'Entre-deux-Mers sont de parfaits représentants des notables ruraux assurant la médiation des pouvoirs princiers et seigneuriaux, comme ceux que Monique Bourin a cernés dans le Languedoc du XIII ${ }^{\mathrm{e}}$ siècle ou que, plus près de nous, Benoît Cursente a identifiés en Gascogne béarnaise et dans la boucle de l'Adour sous l'appellation de pagès ou d' " hommes des casaux ${ }^{23}$. En Entre-deux-Mers, l'enquête révèle trois de leurs attributions, probablement plus larges encore. Ils doivent notamment effectuer la répartition des aubergades demandées traditionnellement par le duc sur l'ensemble des paysans des villae, quelle que soit la condition de ces derniers, avec l'obligation de respecter les immunités des églises. Il leur faut aussi donner conseil et exprimer leur consentement à la nomination des "mandataires ", sorte d'auxiliaires du prévôt ducal agissant dans un ressort déterminé. Enfin, il leur revient de déterminer le montant des amendes pour ceux qui ont refusé de répondre au cri d'appel, Biafora ${ }^{24}$.

Les responsabilités fiscales des prud'hommes les placent en première ligne face aux représentants du roi, prévôt, sous-sénéchaux et sénéchal, qui les sollicitent en priorité pour organiser la répartition de l'effort fiscal entre les habitants des paroisses. C'est pourquoi les prud'hommes de l'Entre-deux-Mers sont particulièrement préoccupés par le dépeuplement de leur région, une inquiétude qui revient fréquemment dans l'enquête comme dans les

\footnotetext{
22 Th. D. HARDY ed., Rotuli litterarum patentium in turri Londinensi asservati, Londres, 1835, vol. I, pars I, 1201-1226, p. 112b (La Souterraine, le 31 mars). Le 16 avril il renouvelle cette confirmation depuis SaintÉmilion, PCSM p. 127; Gallia christiana, t. II, Inst. col. 291.

${ }^{23}$ M. Bourin, Villages médiévaux en Bas-Languedoc, Paris, 1987, t. I p. 321-335, t. II, p. 145-200; B. CURSENTE, Des maisons et des hommes, La Gascogne médiévale (XI'-XV siècle), Toulouse, 1998 ; id., " Le cartulaire du chapitre de Dax et la société des laïcs », dans L'Église et la société dans le diocèse de Dax aux XI XII siècles. Journée d'étude sur le Livre rouge de la cathédrale de Dax, Dax $1^{\text {er }}$ mai 2003, Amis des anciennes églises des Landes \& Comité d'études sur l'histoire et 1'art de la Gascogne, Dax, 2004, pp.73-87. Nous nous permettons de renvoyer à deux articles à paraître sur cette question : F. BOUTOULLE, « Pouvoirs et protagonistes territoriaux dans le domaine ducal gascon : l'Entre-deux-Mers bordelais d'après l'enquête de 1236-1237», dans Les pouvoirs territoriaux en Italie centrale et dans le sud de la France. Hiérarchies, institutions, langages (XII ${ }^{-}$ $X I V^{e}$ siècles), colloque organisé par l'École Française de Rome en collaboration avec l'Università du Firenze et l'Université de Savoie, dir. G. CASTELnUOvo et A . ZORZI, Rome I ${ }^{\mathrm{er}}$ décembre 2006, à paraitre ; ID, « Les élites et le roi : l'enquête de 1236-1237», dans Quand gouverner c'est enquêter. Les pratiques politiques de l'enquête princière. Occident, XIII $-X V^{e}$ siècles, colloque dir. T. PECOUT, UMR Telemme, Aix-Marseille 19-21 mars 2009, à paraître.

${ }^{24}$ PCSM, p. 129, haberet albergagiam in agricolis, villarum forentium cuiuscumque essent agricole et per bonos homines terre albergatores dimidientur, per multas villas et per singulos agricolas (...) et singuli illorum possent comedere semel in anno apud singulos, de illis que agricola comederet et pararet sibi, et debent poni et deponi de consilio et consensu proborum hominum terre (...) Et quicumque non venerit debent talem penam seu gadium qualem statuerit dominus terre, cum probis hominibus terre, quia nulla certa pena super hoc est statuta.
} 
mandements royaux qui tentent de le juguler à leur demande $\left(1214,1219,1222,1233^{25}\right)$ : l'émigration vers Bordeaux ou vers l'Espagne (probablement surestimée) alourdit automatiquement la quote-part de ceux qui restent et ne facilite pas, on s'en doute, le travail de répartition des prud'hommes. Dans une autre partie du domaine ducal, à Labouheyre, dans la Grande Lande, les coutumes données avant 1241 précisent que pour la queste, même si la contribution des habitants de ce bourg castral doit être proportionnelle à leur richesse (« le fort supportant le faible »), l'évolution du nombre de foyers fiscaux ne change rien au montant prévu initialement, poussant la logique du système jusqu'à l'absurde : " s'il n'y avait qu'un seul habitant, celui-ci sera tenu de la payer ${ }^{26}$.

La parole des jurés, telle qu'elle est retranscrite dans l'enquête, jette également de précieux éclairages sur les représentations sociales en vogue dans ce milieu de notables ruraux dont ils se font, d'une certaine manière, les porte-parole. Le procès verbal retranscrit en effet un certain nombre de digressions historiques, tout droit sorties de la parole des jurés, par lequel les élites rurales non nobles cherchent à expliquer l'origine de leur position ou de telle prestation dont ils sont les médiateurs. Cet argumentaire qui leur permet de légitimer leur domination sociale fonctionne ainsi comme une dérivation, pour reprendre un concept de Wilfredo Pareto. La geste carolingienne est captée : interrogés (requisiti) sur les origines de leurs libertés, les jurés répondent que leurs ancêtres, parce qu'ils avaient accompagné Charlemagne dans sa guerre contre les Sarrasins, auraient été gratifiés par la liberté de leurs possessions, en remerciement d'un service gratuit. Une forme de pactisme transparaît aussi d'après l'affirmation que le pouvoir judiciaire aurait été concédé au duc par les justiciables eux-mêmes, pro pace tuenda. Cette vision d'une autorité publique procédant d'une délégation populaire concerne aussi les prérogatives du prévôt et celles du sénéchal en temps de guerre. Attestée également plus au sud dans les fors de Béarn, cette vision constitue un marqueur intéressant de la politisation d'une société rurale dont les élites tiennent à souligner leur engagement volontaire dans la sujétion.

Le procès verbal fait aussi fait état de précédents survenus quelques décennies plus tôt pour expliquer l'origine de telle ou telle prestation. Ainsi, sur l'origine de la queste, l'enquête rapporte pas moins de trois traditions expliquant sa mise en place. Elle est signalée parmi les ressources du comte de Poitiers venant en cette terre à l'appel des prélats dépositaires de « l'affaire de paix et de foi », pour lutter contre les bandes de routiers, mais la faiblesse de cet expédient l'aurait amené à demander l'aubergade. On rapporte plus haut que la queste aurait été assignée à l'archevêque de Bordeaux par Jean sans Terre pour permettre au prélat de nourrir et de vêtir des pauvres, en compensation d'autres rentes que le roi lui avait assignées. Plus loin, il est question d'un précédent remontant à un bailli ducal qui aurait demandé aux hommes de l'Entre-deux-Mers la fourniture d'un cheval de quarante livres, une prestation qui aurait constitué un précédent pour les paroisses ultérieurement astreintes au paiement de la queste. Tous ces thèmes attestent de l'existence auprès des élites rurales d'une forme de

\footnotetext{
${ }^{25}$ Th. D. HARDY éd. op. cit., p. 112 b, PCSM, p. 127. En 1219, il est reproché aux milites d'avoir vendu ou engagé leurs terres ou rentes et de n'être plus capables d'effectuer le servitium debitum pour la défense du pays (Th. RYMER et R. SANDERSON, Foedera, conventiones, literrae et cujuscumque generis acta publica, Londres, 1816-1830, p. 155). Le 4 décembre 1222, le roi enjoint encore aux francs et aux hommes de l'Entre-deux-Mers de revenir sur leurs terres pour lui rendre les coutumes et services dus (Patent rolls of the reign of Henri III preserved in the Public Record Office, Londres, 1901-1913, vol. 1 AD 1216-1225, p. 357). Le 9 août 1233, le roi interdit au maire et à la commune de Bordeaux de recevoir des hommes du roi de l'Entre-deux-Mers cherchant à se soustraire à leur service coutumier (W.-W. SHIRLEY, Royal letters, p. 419-420).

${ }^{26}$ J.-B. MARQUETTE, éd. «Les coutumes de Labouheyre (Herbefauere)", dans Un pays dans sa langue. Le gascon dans l'ensemble d'oc. Actes du colloque de Sabres, sous la présidence de P. BEC, 9-10- octobre 2004, Parc naturel régional des Landes de Gascogne, 2006, pp. 107-140.
} 
mémoire mais aussi d'un degré de culture touchant au politique (l'exploitation de la geste carolingienne) dont on avait jusque là surtout des preuves dans l'aristocratie ou le clergé, mais pas encore auprès des illiterati, peu considérés comme des acteurs culturels. À la manière des communautés religieuses ou urbaines qui servent des objectifs mémoriels en construisant leurs cartulaires, les prud'hommes investissent cette enquête pour y jeter une mémoire sociale en construction.

On remarquera en passant sur quoi s'appuie, chez eux, la conscience de l'injure faite au roi en cette période de progrès de la notion de souveraineté. La référence appuyée au « seigneur-roi » et du service qu'on lui doit montre qu'ils sont imprégnés de la notion de suzeraineté royale et non de celle d'une souveraineté protégée par la lèse majesté que diffusent les légistes du XIII ${ }^{\mathrm{e}}$ siècle ${ }^{27}$. Pour eux, attaquer la dignité du roi est encore perçu comme une violation du serment de fidélité, un parjure d'autant plus grave qu'il concerne quelqu'un de sacré ; mais ce n'est pas encore un crime de lèse-majesté. Pour autant, il n'est pas sûr qu'ils éprouvent pour ce roi distant un respect immodéré. La transmission de la critique par leur canal permet probablement à ces élites rurales d'exprimer par substitution quelques non-dits.

L'enregistrement des propos attribués au sénéchal souligne aussi au moins deux fonctions de l'information au sein des élites rurales. Que ces propos aient été seulement entendus, directement ou indirectement, par des bons hommes nous rappelle qu'au sein de ces communautés d'habitants les individus ont un accès inégal à l'information. Comme dans les villes des derniers siècles du Moyen Âge dont les édiles jaugent, distillent, retiennent ou diffusent l'information au gré des intérêts politiques, la pleine maîtrise de celle-ci est réservée à une élite, en l'occurrence celle qui gère la communauté ${ }^{28}$. En même temps, la dénonciation des exactions peut être vue comme une forme de service qui est rendu au roi au nom des devoirs auxquels sont tenus les sujets, à l'instar des bourgeois de La Réole qui, quelques années plus tôt, dénonçaient dans leurs missives au justicier d'Henri III, Hubert de Bourg, les complots ourdis dans le dos du roi ${ }^{29}$.

Certes, ces notables n'ont pas la force politique que reconnaissent les fors et les chartes de coutumes à leurs homologues du sud de la Gascogne. Il ne leur est pas reconnu, dans la partie de l'enquête qui énumère leurs franchises, de droit à la violence légitime, de juridiction au delà de l'espace domestique ou même un partage de juridiction, comme dans les fors de Bigorre, les coutumes de Corneillan ou celles de Bayonne ${ }^{30}$. Ils ne sont probablement pas aussi redoutables que les valléens du Béarn ou de la Bigorre capables de tenir tête à leur seigneur et de l'empêcher d'entrer dans leurs montagnes. Pour autant, avec leur mémoire construite ou leurs argumentaires rodés, les élites non nobles de l'Entre-deux-Mers s'affichent ou tentent d'adopter la prestance de ceux qui ne courbent pas l'échine.

\footnotetext{
${ }^{27}$ J. HoAREAU-Dodinau, Dieu et le roi : la répression du blasphème et de l'injure au roi à la fin du Moyen Âge, Limoges, 2002.

${ }^{28} \mathrm{Cl}$. GAUVARD, «Introduction», dans Information et société en Occident à la fin du Moyen Âge : actes du colloque international tenu à l'Université du Québec à Montréal et à l'Université d'Ottawa (9-11 mai 2002), s.d. C. Boudreau, K. Fianu, C. Gauvard et al., Paris, 2004, p. 25 ; G. Ferrand, Communautés et insécurité en Rouergue à la fin du Moyen Âge, thèse doctorat Université Toulouse Le Mirail, 2009, p. 305.

${ }^{29}$ W.W. SHIRLEY, Royal letters, p. 50.

${ }^{30}$ H. COUDERC-BARRAUd, La violence l'ordre et la paix. Résoudre les conflit en Gascogne du XI au début du XIII siècle, Toulouse, 2008, p.226-240 ; ID ; «Humbles et violence légale. Quelques cas gascons, XII ${ }^{\mathrm{e}}$-début XIII ${ }^{\mathrm{e}}$ siècle », dans La violence et le judiciaire. Discours, perceptions pratiques, s.d. A. FOLLAIN, B. LEMESLE, M. NASSIET, et alii (dir.), Rennes, 2008, p. 31-46.
} 
Finalement, le cas que nous avons choisi de traiter brièvement ne clarifie pas ce qui fait la rumeur, ce qui distingue ce média fuyant de l'information contrôlée par les autorités. Les propos attribués au sénéchal sont, comme la rumeur, sans origine claire et porteurs d'un fort ressentiment. Ils visent à discréditer, probablement dans le cadre d'une campagne de diffamation. En revanche, ce matériau informatif permet d'en dire plus sur le milieu dans lequel il enfle et que l'on ne peut pas considérer comme une « opinion publique » ou mieux comme un "espace public», au sens habermassien, puisque l'on scrute maintenant ce modèle heuristique en amont du XVIII ${ }^{\mathrm{e}}$ siècle, tout en cherchant à dépasser sa forme et sa définition trop monolithes ${ }^{31}$. Certes, ce groupe de notables ruraux - qui vit, rappelons-le, dans un pays d'habitat dispersé où sont excentrés les lieux centraux dans lesquels s'élabore une sociabilité -, est une source de savoirs collectifs (représentations, informations et rumeurs). D'une certaine manière, ils forment donc un ensemble de personnes privées rassemblées pour discuter des questions d'intérêt commun, ce qui est déjà une part de ce qu'on entend par espace public. Certes aussi, les prises de positions de paysans anglais pendant la guerre civile étudiées par David Carpenter prouvent que la paysannerie de milieu du XIII ${ }^{\mathrm{e}} \mathrm{s}$. est consciente des enjeux politiques du temps et traversée de débats passionnés ${ }^{32}$. D'une certaine manière, cet espace-là est plus autonome et moins verticalement structuré que «l'espace public de la représentation » caractéristique des sociétés médiévales selon Jurgen Habermas. Pour autant, les matériaux informatifs recueillis par l'enquête émanant du groupe qui nous intéresse, qu'il s'agisse des représentations historico-politiques ou de ce que la rumeur attribue au sénéchal, ont tous la même vocation : il s'agit moins de critiquer le roi, de manière plus ou moins rationnelle, que de légitimer la domination sociale des prud'hommes. L'enjeu est de défendre la posture traditionnelle que leur confère la médiation du dominium face à la concurrence des baillis. La circulation de ce type d'information ne révèle donc pas un espace public, mais plutôt ce qu'on pourrait appeler un espace communautaire de légitimation.

Frédéric Boutoulle

Université de Bordeaux-UMR Ausonius 5607

\footnotetext{
${ }^{31}$ S. HABER, art. cit.

${ }^{32}$ D. CARPEnTER, « English Peasant in Politics 1258-1267 », dans Id., The Reign of Henry III, Londres, 1996, p. 309-348.
} 